\title{
Las políticas de inserción estratégica de los países del golfo Pérsico en el cuerno de África
}

\section{Ricardo Agustín Benítez}

\section{RESUMEN}

El presente trabajo busca abordar el sistema de inserción estratégica de los países del golfo Pérsico en el cuerno de África, mediante un enfoque multidimensional que permita entender cómo dichas estrategias son flexibles y pragmáticas, en tanto dependen, en gran medida, de la cooperación o resistencia de los actores que se encuentran involucrados. Los países africanos son actores activos en el proceso de inserción estratégica de Estados extra-regionales con intereses en la zona, por lo que pueden definir los alcances de las políticas aplicadas, adquiriendo un mayor margen de negociación, en la medida que varias políticas de inserción se focalizan en temas o puntos geográficos específicos. Analizar cómo se transforman las relaciones entre estos actores, permite reflexionar y visibilizar el rol que tienen los Estados africanos para condicionar las políticas de influencia de actores extra-regionales.

Palabras claves: Estrategia, competencia, rivalidad, conflicto.

\section{STRATEGIC INSERTION POLICIES ON THE HORN OF AFRICA}

\section{Cooperation and resilience among Persian gulf's countries and Horn of Africa States}

\begin{abstract}
The article provides a multidimensional approach of the strategic insertion policies of extra-regional states on the Horn of Africa. It
\end{abstract}

\footnotetext{
* Magíster en relaciones internacionales, Universidad del Salvador. Buenos Aires (Argentina). Director de la revista Ágora Internacional de la Asociación para las Naciones Unidas de la República Argentina (ANU-AR). Coordinador del área de investigación "Explorando África", del Centro de Estudios de Política Internacional de la Universidad de Buenos Aires (Cepi-uba) [rbenitez@anu-ar.org]; [https://orcid.org/0000-0002-3902-2288].

Recibido: 11 de enero de 2021 / Modificado: 5 de marzo de 2021 / Aceptado: 9 de marzo de 2021

Para citar este artículo:

Benítez, R. A. (2021). Las políticas de inserción estratégica de los países del golfo Pérsico en el cuerno de África. OASIS, 34, pp. 87-105

DoI: https://doi.org/10.18601/16577558.n34.06
} 
seeks to provide a better understanding about how these policies are flexible and pragmatic considering the degrees of dependency on cooperation and resistance of the States are focused on. African countries played an active role in the strategic insertion process of extraregional states because they could define the frame of application of these policies while they acquire a better space of autonomy. The article will highlight how these policies, when focused on specific issues, demonstrate improvement in the bargaining capacity of African states. Analyzing the dynamics between these actors will provide a new understanding about how African States are key players shaping the politics of extra-regional actors when these are oriented to influencing their local scenarios.

Key Words: Strategy, competence, rivalry, conflict.

\section{INTRODUCCIÓN}

Durante los últimos 20 años se ha ido perfilando un nuevo proceso de ordenamiento de poder a nivel global. La consolidación de un orden multipolar, el surgimiento de nuevos actores con influencia global y regional, como Rusia, China y Turquía, entre otros, y la diversificación de los instrumentos de poder geopolítico permitieron el desarrollo de nuevas dinámicas de interacción internacional definidas por la competencia, la cooperación, la influencia y el posicionamiento estratégico en nuevos escenarios.

El continente africano es un escenario, en donde la proyección de influencia de actores extra-continentales ha alcanzado un nivel de relevancia internacional considerable. El valor comercial, el potencial económico, su vinculación con las rutas comerciales globales y la existencia de vastos recursos estratégicos, han fomentado el interés de varios actores internacionales con diversos grados de poder tales como Emiratos Árabes Unidos (en adelante EAU) y Rusia, los cuales fundamentan la necesidad de desarrollar una política de inserción en el teatro africano (Ouma, 2012, p. 836).

Es importante aclarar que el presente trabajo se enmarca en una lectura de planificación estratégica en donde los intereses de los agentes implicados se configuran en función de maximizar su poder mediante la consecución de objetivos proyectados como necesarios para tal fin (Isern, 2021). En este sentido, la maximización del poder mencionada propone una dinámica de rivalidad donde la consecución de un objetivo de un actor puede ser identificada por otro como en detrimento de su posición, la cual debe contrarrestar o balancear.

Por otro lado, existe un marco de análisis en el que el escenario adquiere preponderancia y donde la geografía se convierte en un elemento de análisis. Las características puntuales de la región del cuerno de África, que atraen el interés de los actores en el escenario, representan un factor clave que se debe considerar. No obstante, las políticas se construyen en función del escenario y no a causa de este, por lo que el aspecto geográfico y geopolítico no representa un marco teórico para entender la conducta de los Estados o sus políticas exteriores (Scholvin, 2016, p. 4).

Por lo tanto, a lo largo del trabajo remarcaremos la construcción de políticas y toma de decisión en función del accionar de los agentes que interactúan en un escenario geográfico 
específico con la finalidad de describir y explicar, de forma ordenada, como este entramado de políticas define un escenario de acción en el que todos los agentes implicados tienen relevancia.

Precisando el enfoque de análisis, la región del cuerno de África (en adelante $\mathrm{CdA}$ ) se caracteriza por ser un área donde la intensidad de este fenómeno de inserción es muy visible y variada. Su posicionamiento estratégico en cercanía a rutas comerciales importantes, la inestabilidad política, las amenazas de seguridad presentes y la convergencia de los intereses de actores internacionales con diferentes niveles de poder, dan forma a un escenario con múltiples desafíos, variables de análisis e intereses contrapuestos, que permiten visualizar la existencia de un nuevo patrón de inserción geoestratégica (Iss, 2019).

Este nuevo patrón que observamos en la región se expresa en la implementación de un conjunto de políticas que combinan, estructuran y priorizan, en diferentes grados, los siguientes temas (Leboeuf, 2019, p. 21):

a. La influencia en finanzas mediante acceso a créditos, préstamos y ayudas económicas.

b. El despliegue de esfuerzos diplomáticos vinculados a mejorar la confianza, la cooperación y la resolución en conflictos no resueltos.

c. La construcción de infraestructuras de uso dual (civil-militar), vinculadas al comercio (puertos y aeropuertos) transportes (autopistas, vías férreas) a la industria (centrales energéticas, conectividad) y producción (em- presas, zonas económicas exclusivas, servicios) es otro elemento sustancial de cooperación y competencia.

d. La instrumentalización de elementos de soft power vinculados a programas de capacitación técnica, cobertura mediática, educación, turismo, idiomas y caridad.

e. La consolidación de un componente securitario orientado a la cooperación militar, la capacitación de personal, la asistencia militar, y la presencia permanente de efectivos militares en bases navales, terrestres y áreas construidas en la región ${ }^{1}$.

Las políticas utilizadas para tal fin no deben ser analizadas como un conjunto coherente y racional diseñado para su implementación estricta. Dada la elevada volatilidad del escenario, muchas de estas políticas varían con el contexto y se adaptan a los nuevos desafíos planteados en el tiempo. A su vez, es importante destacar que las políticas de inserción también responden al equilibrio de fuerzas existentes al interior de cada Estado, representado en la competencia burocrática interna, la cual define un mayor o menor alcance de cada una de estas políticas estratégicas (Leboeuf, 2019, p. 10).

Este trabajo buscará explicar las "políticas de inserción estratégica" entendidas como aquellas políticas que buscan garantizar posiciones ventajosas a los países del golfo Pérsico en la región específica del CdA, analizando la importancia de esta región como caso de estudio y puntualizando en los diversos tipos de

$1 \quad$ La cursiva es propia para resaltar el concepto. 
actores que actúan en el mismo, promoviendo un reconocimiento de las relaciones entre los países del golfo y los del CdA. A su vez, observaremos cómo los actores, en sus interacciones, interpretan, priorizan y se vinculan con la gestación de un contexto favorable o limitado de estas políticas de inserción, dando forma a un escenario volátil, cambiante y de compleja interrelación en donde los Estados del CdA juegan un rol activo y que condiciona los intereses de los actores extra-regionales.

Por lo tanto, este trabajo realizará un análisis de las políticas estratégicas entendidas en este formato, comprendiendo que el éxito o fracaso en la implementación de las políticas no responde solo a los Estados que la planifican con el objetivo de influir en una región, sino que deben comprender los intereses y los efectos de estas en el Estado receptor, elemento que definirá, en última instancia, el nivel de aceptación o resistencia a las relaciones entre ambos Estados.

\section{EL ESCENARIO, SUS ACTORES E INTERESES}

\section{El cuerno de África}

$\mathrm{Al}$ analizar la región del cuerno de África, se destacan tres elementos que definen su importancia a nivel global. En primer lugar, debemos mencionar su posición geográfica en la cercanía a las rutas comerciales localizadas en el mar Rojo, el acceso a pasos marítimos como el estrecho de Bab el Mandeb y el canal de Suez que vinculan el océano Índico y el mar Mediterráneo, y que representan la importancia del espacio marítimo y su influencia, en una región conocida como clave para el suministro energético global de petróleo y gas (Neil, 2019).

En segundo lugar, la diversidad de actores que proyectan sus intereses en la región se ha multiplicado en los últimos tiempos. En ella, se observa la presencia de potencias globales como China, los EE. UU., Francia y potencias regionales como Irán, Turquía, Arabia Saudita o EAU sumado a los propios actores locales de la ribera africana.

En tercer lugar, la complejidad estratégica, producto de la interacción entre un elevado número de actores, intereses diversos en un área estrecha y definida por la una limitada institucionalidad regional $^{2}$, la existencia de conflictos militares como los casos de Yemen y Sudán del Sur, la rivalidad diplomática entre los países del golfo Pérsico y entre Arabia Saudita e Irán, al igual que la inestabilidad política en Sudán, Eritrea, Etiopía, son elementos que, sumados a la actividad terrorista de Al-Shabbab en Somalia y Al-Qaeda en Yemen y varias disputas territoriales aún sin definir, establecen un escenario con amplios niveles de competición, rivalidad y carencias

2 A nivel regional, opera el IGAD (Intergovernmental Authority of Development) como organismo regional que aglutina a los Estados del cuerno de África con estrategias de cooperación e integración. En algunos Estados de la región se yuxtaponen otras instituciones tales como coMESA (Common Market for Eastern and Southern Africa), cen-sad (Community of Sahel-Saharan States). Para más información ver: European Center for Development Policy Managment. Disponible en https://indd.adobe.com/view/f49ac87d-7aa3-4cf7-822e-841d674bbc92 
de cooperación, entrelazados con el traspaso de nuevos patrones de rivalidad extra-regionales (Mahmood, 2019).

\section{Los Estados del golfo}

$\mathrm{Al}$ abordar la temática de inserción estratégica en el CdA, el énfasis suele orientarse a la política de grandes actores globales como China, Unión Europea, EE. UU. o de Rusia en un contexto general (Ursu \& Van den Berg, 2018).

Un segundo nivel de análisis suele enmarcarse en la creciente participación de los países del golfo Pérsico en la región. Varios trabajos al respecto (Lefebvre, 2012; Leboeuf, 2016; Marchal, 2018; Mahmmod, 2019) por mencionar algunos, destacan la traslación de las dinámicas competitivas de las potencias de Oriente Medio a la región del CdA, La "traslación de conflictividad", como preferimos mencionarla, implicaría la exportación de intereses basados en hipótesis de conflicto o de rivalidad creadas en un escenario local-como ejemplo el Medio Oriente- y que son reproducidas por los mismos actores en escenarios extra-regionales como puede ser el CdA en este caso.

En referencia a lo anterior, el presente trabajo buscará incluir entre los denominados "Estados del golfo" a las políticas de Turquía y a la presencia de Irán en la región, dado que enfatizan la política más activa de Arabia Saudita, EAU y Qatar como actores que promueven nuevas dinámicas de influencia en el CdA.

Un elemento distintivo de estos actores consiste en la creciente competencia por influencia y poder a consecuencia del contexto de inestabilidad y conflicto creciente en su región cercana. Estos procesos se explican por la inestabilidad institucional originaria de las "primaveras árabes" y los posicionamientos rivales respecto al nuevo escenario, los efectos de la guerra en Yemen, la rivalidad ideológica entre Arabia Saudita e Irán y la política más activa de Turquía como nuevo referente regional (Marchal, 2018).

La complejidad de estas rivalidades, son trasladadas a los países del CdA mediante la búsqueda de nuevos aliados, el acceso a nuevos espacios de poder y el prestigio de contar con elementos de negociación contra sus rivales, exportando una dinámica de competencia y suma cero ${ }^{3}$ a una región históricamente inestable. Al respecto, las relaciones entre los Estados del CdA y los de golfo se definen como asimétricas en favor de estos últimos, debido a sus mayores capacidades económicas, financieras, de influencia política y de autonomía diplomática y militar (ICG, 2019).

$\mathrm{Al}$ analizar las relaciones golfo-cuerno de África, los beneficios se visualizan para los

3 En relaciones internacionales, la dinámica de suma cero refiere a la teoría de los juegos en donde las ganancias acumuladas de un actor son iguales a la suma de las pérdidas del otro actor rival. En este aspecto, y como ejemplo de la región, un acceso preferencial a una concesión portuaria por parte de un actor extra-regional restringe indefectiblemente el acceso a los otros actores al recurso y garantiza un elemento de poder que lo diferenciará del resto de los actores implicados en el área. Isern, P. (2021) Relaciones Internacionales y juegos de suma cero, Fundación ICBC. Disponible en https://www.biblioteca.fundacionicbc.edu.ar/index.php/Relaciones_Internacionales_y_juegos_de_suma_cero 
últimos a través de las potencialidades para el desarrollo económico debido a la promoción del comercio, el mayor acceso a inversiones y la creación de infraestructuras. A su vez, el comercio funciona como motor de la confianza y la cooperación como elemento central para la resolución de conflictos (Woldemichael \& Andemariam, 2019). Sin embargo, la competencia por el acceso privilegiado a mercados y la lógica de suma cero que exportan varios actores se entiende como un nuevo factor de inestabilidad al largo plazo debido a la creación de esferas de influencia vinculadas a conflictos extra-regionales, sumando nuevos ejes de conflicto a los ya existentes localmente, proceso que reconoceremos como "traslación de conflictividad".

\section{Los Estados del cuerno de África}

Para identificar los Estados ubicados en el cuerno de África es necesario empezar por precisar los límites de la región en cuestión. Dependiendo del tipo de análisis y el autor, la extensión de la región puede variar desde cuatro hasta ocho Estados africanos. Sin embargo, en términos geográficos se entiende que es la región terrestre ubicada entre la desembocadura sur del mar Rojo, la punta oriental del golfo de Adén y la zona costera del océano Índico hasta la línea del Ecuador (Encylopedia Britannica, 2021).

En términos políticos los Estados centrales de la región se pueden identificar en los siguientes: Djibouti, Eritrea, Etiopía, Somalia. Sin embargo, a nivel multilateral, las cuestiones sociales, securitarias, diplomáticas y contextuales requieren de un sistema político regional que se extiende a varios países cercanos a este núcleo primario por lo que se incluye a Sudán, Sudán del Sur, Kenya y Uganda (IGAD, 2019).

A su vez, en términos estratégicos, la región del CdA incluye a los países de la península arábiga, a aquellos ribereños en el mar Rojo y otros actores con presencia permanente en la región, ampliando el escenario los siguientes Estados: Egipto, Israel, Arabia Saudita, Yemen, Omán, EAu, Qatar, Turquía, Irán, China, Japón, Francia, UE, Italia, EE. UU., Rusia.

En el siguiente trabajo, para analizar las relaciones de los Estados del golfo y del CdA adoptaremos una lectura estratégica. Para los Estados del golfo solo incluiremos a Arabia Saudita, EAU, Qatar e Irán junto a Turquía como potencia extra-regional con intereses en la región, ya que son los casos más paradigmáticos para analizar y para los Estados del CdA seleccionaremos a aquellos Estados que tienen acceso al espacio litoral regional o dependen de este como en el caso de Etiopía, por lo que Estados como Kenia, Sudán del Sur y Uganda quedarán relegados del análisis.

En relación con los aspectos securitarios, la región ha sido fuente de fuertes rivalidades vinculadas a la definición de límites territoriales y en donde se han registrado conflictos armados hasta la actualidad, siendo destacable la situación de Sudán del Sur y Somalia. En este último caso, la presencia de la milicia islamista Al-Shabbab se ha identificado como uno de los principales problemas para la situación securitaria y el desarrollo regional.

A su vez, en el último lustro se ha destacado el accionar de los Estados del CdA para mejorar la seguridad territorial, no ausentes de reveses, por medio de la celebración de acuerdos limítrofes con la mediación de ac- 
tores extra-regionales e instituciones internacionales, así fueron los casos entre Eritrea y Djibouti, Eritrea y Etiopía, el arbitraje por los límites marítimos entre Kenia y Somalia por la prospección petrolera, entre los principales.

La promoción de la cooperación y mantenimiento de la seguridad institucional en países vecinos es otra cuestión a destacar. En este sentido, es notable el despliegue de la misión de la АмІsом (African Union Mission in Somalia) supervisada por la Unión Africana y compuesta por unidades completamente africanas, siendo la acción de Kenia clave para su realización. Por otro lado, Etiopía se ha mostrado como un actor importante a nivel regional, gracias a su influencia diplomática y su geolocalización central en la región, actuando como mediador en el conflicto de Sudán del Sur y en la política de transición de Sudán o en el control de las fronteras somalíes.

Respecto de la institucionalidad regional, consideramos que corresponde mencionar en primer lugar al denominado Intergovernmental Authority on Development (en adelante IGAD) con oficinas centrales en Djibouti. Creada en 1996, tiene como objetivo reforzar la cooperación en materia de seguridad alimentaria, un problema crónico en la región, la protección ambiental, la cooperación económica, la integración regional y el desarrollo social, de la paz y la seguridad. Entiende que mediante la cooperación e integración se promueve el desarrollo de la paz y la seguridad entre los Estados regionales (IGAD, 2019).

Un segundo elemento para destacar es la firma de los Estados del CdA del Africa Continental Free Trade Area (AfCFTA, en sus siglas en inglés), organismo que promueve la creación de una zona de libre comercio a nivel continental. Aunque el proceso es ambicioso, deja en claro el reconocimiento de las potencialidades africanas desde un punto de vista local y la necesidad de ampliar dichas capacidades en un espacio de comercio común. Se ha observado que uno de los requisitos primordiales para el establecimiento de este proyecto descansa en la conformación de mecanismos de cooperación que permitan vincular redes de comercio a nivel intra-regional (Iris, 2017). Este requisito impone la necesidad de resolver cuestiones limítrofes, disminuir la potencialidad del conflicto y fomentar la confianza entre un conjunto de actores que históricamente han sido rivales.

Un tercer elemento que ha dado impulso a las políticas de cooperación regional proviene de la influencia extra-regional. En particular, algunos Estados del golfo han promovido y apoyado la celebración de reuniones para resolver disputas limítrofes vinculadas a los intereses de mejorar las condiciones de seguridad, como ha sido el caso del apoyo de EAU al acuerdo entre Etiopía y Eritrea. A su vez, los EE. UU. son otro elemento clave para la consolidación de la seguridad en Somalia y cooperan financieramente e institucionalmente con el gobierno local y organismos regionales para fomentar la estabilidad del país (Usaid, 2021).

\section{LAS ESTRATEGIAS DE INSERCIÓN EN EL CUERNO DE ÁFRICA}

\section{Estrategia de inserción desde los países del golfo}

Podemos clasificar las políticas de inserción en 5 grandes áreas: Finanzas, infraestructuras, 
diplomáticas, cooperación y securitarias. Cada una de estas políticas estará definida por las capacidades de cada actor y sus intereses especiales, mientras que su despliegue y efectividad dependerá del contexto y de la interacción con el Estado receptor y los Estados rivales con intereses en la misma área.

A inicios del siglo xxI, los países del golfo Pérsico, incluyendo a Irán y Turquía, mantenían diferentes grados de presencia e influencia en la región del CdA. Eran destacables las buenas relaciones entre Irán y Sudán mediante la cooperación económica y militar, producto del aislacionismo diplomático de ambos países. En la misma línea, en 2008, Eritrea le permite a Irán el acceso al puerto de Assab. Estos vínculos, le permitían a Irán tener acceso a puertos de suministro sobre la costa meridional israelí y saudí, históricos rivales regionales de este Estado (Lefebvre, 2012).

A su vez, Turquía fue reorientando su política exterior durante el primer decenio hacia su región oriental. El gobierno del Adalet ve Kalkınma Partisi (en adelante AKP) decidió priorizar una nueva política basada en el prestigio, crecimiento económico y construcción de liderazgo regional (Asya, 2019). En el CdA su política de inserción se sustentó en políticas económicas y financieras (Gurbuz, 2018). Su principal socio se encuentra en Somalia, donde el comercio bilateral ha superado los USD 100 millones en 2016 y en donde ha establecido una base militar. Por otro lado, posee acuerdos de construcción de infraestructuras en Etiopía, Sudán, principalmente en la zona del puerto de Suakin, y en Djibouti donde además de una zona franca también se percibe la construcción de una nueva base militar.

Arabia Saudita ha capitalizado su posición en el CdA a partir de 2015, donde la coyuntura política de la familia real y el ascenso de nuevas figuras políticas, promovieron la adopción de una política de liderazgo regional, impulsada de forma exponencial por los efectos de las denominadas "primaveras árabes" y el aumento de la competencia estratégica con Irán. Para contrarrestar esta última ha promovido la asistencia a gobiernos o facciones con personalidades fuertes, vinculadas al sector militar como en Egipto o Sudán. La guerra en Yemen y la necesidad de recurrir a nuevos aliados, es el segundo elemento que modificó la política saudí en el CdA, atrayendo a aliados iraníes como Eritrea y Sudán a su esfera de influencia. Por último, el empoderamiento de Arabia Saudita como un líder regional y global se expresa en los avances sobre la seguridad regional en el CdA, tomando como casos la mediación en las relaciones entre Djibouti y Eritrea, y entre este y Etiopía, al igual que en proyectos como el Red Sea Forum for Coastal States que busca generar un espacio de coordinación entre los países de la cuenca del mar Rojo ${ }^{4}$ y que proyecta un mayor control saudí de la región de la cuenca del Nilo (ICG, 2019).

\footnotetext{
4 Actualmente, faltan países de la cuenca del mar Rojo por incorporarse. En el caso de Eritrea, esta aún no participa y, a su vez, se está considerando una potencial incorporación de Etiopía.
} 
Uno de los actores más prominentes en la región es Emiratos Árabes Unidos. Afectado por la dinámica regional, ha impulsado una política securitaria que se sustentaría en las buenas relaciones con países amigos, evitar las insurrecciones sociales que generen inestabilidad política y disminuir la injerencia de Irán en la región. Para ello ha desplegado una política que incluye inversiones, desarrollo comercial e instalaciones de bases militares a lo largo de la costa del CdA, siendo Eritrea y la región de Somalilandia sus principales puntos de referencia. Su política de intervención selectiva en las regiones de Somalia es un elemento que afecta los intereses de unificación del gobierno central de ese país (Mesfin, 2019).

Por último, podemos mencionar a Qatar, quien han promovido una política basada en la influencia mediática a través de su red AlJazeera y comercial, mediante la promoción de inversiones. Las coberturas mediáticas de los procesos de las revueltas sociales de 2011 fueron claves para contextualizar el devenir de las manifestaciones. Sin embargo, ha tenido que reestructurar su actividad en la región como respuesta al bloqueo diplomático de Arabia Saudita y sus aliados en 2017. Promoviendo una diversificación de sus relaciones diplomáticas, ha encontrado en la región de $\mathrm{CdA}$, un terreno fértil para mejorar sus inversiones y aumentar sus aliados, reduciendo los efectos del bloqueo. Su nivel de inserción es menor a la del resto, pero la posición de AlJaazera, como uno de los principales medios de comunicación regionales, le ofrece una herramienta de soft power única, al tiempo que su alianza con Turquía, le habilita el acceso a mercados estratégicos con condiciones beneficiosas como es el caso de Somalia (ICG, 2019).

\section{La dinámica de competencia de los países del golfo en la región}

Las primaveras árabes y las revueltas sociales desataron un período de inestabilidad política en Medio Oriente, produciendo nuevas alianzas y rivalidades en la región, con una gran durabilidad en el tiempo. La posibilidad de establecer nuevos modelos políticos para los países en rebelión fue objeto de fuertes influencias de estrategias rivales como son la saudí y la turca, al igual que permitió una mayor presencia de la influencia iraní a través del conflicto en Siria.

Dicha competitividad vinculada al liderazgo regional se acrecentó debido a la guerra en Yemen de 2015, estableciendo un escenario de conflicto en el centro del eje golfo Pérsicocuerno de África, trasladando las dinámicas estratégicas de Medio Oriente a una nueva región periférica.

A su vez, el 2015 se destacó por la decisión de la República Popular China (en adelante Rpch) de establecer su primera base militar en la región, situada en Djibouti, secundada por la realización de una primera misión de rescate de nacionales chinos de Yemen por vía marítima. Al mismo tiempo, este año significó el inicio de construcciones destinadas a la formación del proyecto Belt and Road Initiative (BRI) en el continente, con un énfasis crucial en la región del CdA (Lin, 2015).

A pesar de que la política de Rpch en el CdA está vinculada a intereses económicos, su presencia aumentó su influencia política y su 
participación en tareas de seguridad, incrementando el interés de EE. UU., la UE y de actores secundarios como los países del golfo, quienes reconocieron la mayor necesidad de ampliar su presencia en la región.

En 2011 se realizó la primera visita de Recep Tayyip Erdogan, presidente de Turquía a Somalia en promoción de mejorar las relaciones bilaterales y la búsqueda de inversiones. Ya en 2014, este país ofrece la concesión portuaria de Mogadiscio a una empresa turca (Gulf Bussines, 2018).

En marzo de 2015, Arabia Saudita realizó un acercamiento con Sudán por medio de apoyos económicos y ayuda financiera, rápidamente aceptadas por un gobierno limitado por las sanciones económicas y el aislacionismo diplomático. Es notable que Sudán es un país estratégico en tanto posee un litoral extenso y contiguo del mar Rojo enfrente a las costas saudíes y mantenía una alianza económica y militar con la República Islámica de Irán. Las negociaciones incluyeron una ayuda económica a cambio del apoyo militar a la coalición militar de los saudíes contra los rebeldes hutíes yemeníes respaldados por Irán (ICG, 2019). Sudán abandona su alianza con Teherán por una cooperación más beneficiosa para superar sus dificultades económicas y securitarias facilitando la política de Riad. En el nuevo contexto revolucionario del país, el gobierno de transición ha promovido una consolidación de la democracia y buena vecindad para reducir el peso de las sanciones económicas, aunque ha declarado retirar las tropas de Yemen, los condicionamientos económicos podrían hacer que su permanencia perdurase a cambio de nuevos apoyos (Marchal, 2020)
Al mes siguiente, Djibouti revoca el acuerdo de concesión portuaria otorgado a DP World, la empresa contratista emiratí para comercio y puertos. Dicha concesión había sido otorgada en 2006 y la decisión irrumpía unilateralmente el contrato en detrimento de los intereses emiratíes en el país. A su vez, en el marco de la organización de las operaciones militares contra Yemen, Arabia Saudita firma un acuerdo de cooperación militar con Eritrea, asegurándose aliados en todo su flanco sur y desplazando a los iraníes de nuevo, quienes tenían un acceso privilegiado al puerto eritreo de Assab desde 2008.

En el 2016, la tensión entre los saudíes e Irán se recrudece ante la ejecución de un imán chií en el reino y la reacción en Teherán de rechazo mediante los ataques a la embajada de Arabia Saudita en esa ciudad. Sudán, Djibouti, Eritrea en coordinación con Arabia Saudita condenan a Irán por los incidentes. Somalia se une a las condenas a Irán al tiempo que se anuncia la entrega de unos 50 millones de USD en ayuda financiera a ese país (Reuters, 2017).

Somalia es un actor particular, su débil Estado central y su estructura federal permiten la penetración de intereses extranjeros en distintas partes del territorio. Ejemplos puntuales son los relacionados con las regiones de Somalilandia y Puntland, quienes actúan como gobiernos autónomos celebrando acuerdos con otros Estados. En septiembre del 2016, las autoridades de Somalilandia acordaron la concesión portuaria de Berbera por un plazo de 30 años a DP World, la empresa emiratí que había perdido la concesión del puerto de Doraleh el año anterior, a solo $250 \mathrm{~km}$ al 
oeste del nuevo puerto. A su vez, el acuerdo comprendía la creación de una base militar de EAU en la zona.

En abril de 2017, el gobierno de Puntland acuerda una nueva concesión a los emiratíes, esta vez del puerto de Bosaso, $460 \mathrm{~km}$ al este de Berbera. En el mismo mes, Arabia Saudita y Djibouti firman un acuerdo de cooperación militar contemplando la construcción de una base militar saudí. Considerando la alianza militar con los EAU en la guerra en Yemen, dicha concesión permitiría el control del bloque liderado por Riad de los principales puertos ubicados entre Eritrea y la salida del golfo de Adén, bloqueando el acceso naval iraní en toda la región.

En 2017, la política de rivalidades en el golfo Pérsico y Medio Oriente pone en conflicto los intereses de Arabia Saudita con los de Qatar. Esta última es objeto de un bloqueo comercial que le obliga a reestructurar sus relaciones diplomáticas y comerciales. Aunque reticente a un acercamiento con los iraníes, el gobierno qatarí ha estrechado lazos con Turquía, Estado que mantiene sus diferencias con los saudíes en el escenario de Medio Oriente (Skinner, 2021).

Qatar mantenía buenos lazos históricos en la región del CdA, ya en 2010 había oficiado como mediador entre Eritrea y Djibouti, por un desacuerdo fronterizo. A su vez, la cadena televisiva Al Jazzera posee una influencia notable allí y es considerada uno de los principales recursos de influencia de Qatar. Dado el reciente alineamiento de Eritrea y Djibouti con Arabia Saudita, estos apoyaron el bloqueo de este país contra Qatar a pesar del historial de cooperación entre ellos (ICG, 2019).
Para septiembre de 2017, Turquía inauguró su primera base militar en África ubicada en Mogadiscio, solo a 3 años de la concesión portuaria del mismo sitio. El gobierno turco hizo de Somalia uno de sus principales puntos de inserción estratégica, el apoyo al gobierno central y el fortalecimiento de sus infraestructuras, ayuda financiera y comercial, junto a emprendimientos culturales y securitarios ha definido la agenda entre ambos actores (Asya, 2019). Se debe considerar que, en el escenario de Medio Oriente, los intereses turcos y saudíes se encuentran en competencia y, en este sentido, el reforzamiento del gobierno central somalí, debilita la capacidad de inserción de los EAU, un aliado estratégico saudí y principal soporte financiero y diplomático del gobierno de Somalilandia (Mahmood, 2019).

En diciembre de 2017, Erdogan visitó Sudan y firmó un acuerdo para invertir en proyectos de mejora del puerto de Suakin por un plazo de 99 años. Siendo puerto Sudán el principal en el país, Suakin es un emprendimiento de largo plazo que requiere de una gran cantidad de inversiones. Sin embargo, el interés de Turquía de reforzar su posición en el mar Rojo representa una potencial amenaza al sistema de alianzas ofrecido por los saudíes en toda la región. Turquía ofrece un modelo de cooperación menos condicionante en lo securitario y más vinculado con el desarrollo económico y el fortalecimiento institucional. En este aspecto, el interés en Suakin excede la dimensión estrictamente militar y se sustenta en el desarrollo de todo el ambiente portuario y urbano (Amin, 2018).

En abril de 2018 Somalia confiscó UsD 9,6 millones de un avión de EAU, por lo que 
este reduce su cooperación militar a Mogadiscio. Este hecho sucedió al año de la concesión portuaria del puerto de Puntland, Bosaso, a una compañía emiratí.

El 15 de junio de 2018, los EAU aumentan su inversión en Etiopía y se ofrecen de mediadores en el acuerdo Etiopía-Eritrea. Las inversiones suman los 3 billones de USD entre diferentes proyectos y destinan 1 billón para las reservas nacionales. Este nuevo interés se enmarca en el ascenso de una nueva figura política en el país Abiy Ahmed, quien asumió como primer ministro el 2 de abril de 2018 y quien impulsó un programa de reformas políticas y económicas que buscan perfilar al país como un nuevo actor estabilizador en la región, mediante el aumento de la cooperación, la multilateralidad, la mediación en conflictos y la pacificación de diferendos, que inició por la solución del conflicto fronterizo con Eritrea-antigua región etíope hasta 1993-actual aliado de los EAU.

En septiembre, Djibouti nacionaliza el puerto de Doraleh en contra de la empresa DP World de EAU aumentando el nivel de enfrentamiento con Dubai, el caso fue llevado a la London Court of International Arbitration quien falló a favor de DP World (The Maritime Executive, 2019). Se debe destacar que, en enero del mismo año, se inauguró la vía férrea que conecta Addis Abeba, en Etiopía, con la ciudad de Djibouti. El 90\% del comercio exterior de este país sale por el puerto de Doraleh, las inversiones de ambos tramos fueron en total capitales chinos, lo que explicaría la necesidad de aumentar el control nacional del principal y único puerto nacional.
Aunque Djibouti se perfila contrario a los intereses emiratíes, sus niveles de acercamiento con Arabia Saudita permanecen inalterados, incluso, estos han adoptado el papel de mediadores y hospedan a los gobiernos de Eritrea y Djibouti en las rondas de negociaciones sobre acuerdos limítrofes, acordados antiguamente bajo el auspicio qatarí.

En diciembre, Qatar y Somalia firman acuerdos bilaterales que incluyen la construcción del puerto de Hobyo y algunos memorandos de entendimiento en materia de economía, comercio y políticas fiscales. $\mathrm{Al}$ ser Qatar un aliado estratégico de Turquía, sus accesos a acuerdos preferenciales con Somalia podrían ser explicados desde el aspecto de complementariedad comercial (Van den Berg \& Meester, 2019).

En el transcurso del 2019, los lineamientos estratégicos en la región han sido afectados por la revolución en Sudán. La conjunción de conflictos armados en varias regiones, un sistema de gobierno clientelista y militarizado, y una crisis inflacionaria y de endeudamiento en un país extremadamente pobre, llevó a la población civil a salir a las calles y presentar sus demandas que finalizaron con el golpe de Estado de las fuerzas militares en abril, con lo que se dio fin al régimen de Omar al-Bachir (Iss, 2019).

La modificación de un cambio de poder entre las élites sudanesas representaría una amenaza a los intereses securitarios creados por los saudíes en el país. Los sectores militares en los que se apoyaba el gobierno de Omar alBachir continúan en el poder compartiéndolo con el sector civil. La transición democrática podría reducir aún más la influencia de los 
militares, aliados tradicionales de los saudíes quienes, mediante el apoyo económico, clave para el éxito de la transición, buscarían consolidar su influencia en la política interna del país (Woldemichael, 2020).

Por otro lado, los sectores civiles aglutinan organizaciones laicas e islamistas que son apoyados por Turquía y la UE, quienes promueven la búsqueda de un nuevo sistema político superador del gobierno militar. A su vez, Etiopía sigue de cerca el proceso, interesado en mejorar las relaciones con Sudán para garantizar su proyecto energético más importante, la represa del renacimiento en la cuenta del Nilo Azul y que genera tensiones con Egipto, aliado de Arabia Saudita. La obra, representa un potencial energético vital para el crecimiento industrial y, a su vez, asegura un instrumento de poder de negociación clave a nivel regional, por ello, la política de Etiopía y de Sudán es seguida muy de cerca por los saudíes y EAU dado que afecta directamente sus esquemas estratégicos en el CdA.

A lo largo de este apartado se ha podido apreciar la necesidad de adaptación de las estrategias de los Estados del golfo en relación con sus rivalidades con otros actores. Lo que denominamos traslación de conflictividad implica la exportación de intereses basados en hipótesis de conflicto o de rivalidad creadas en escenarios extra-africanos en los Estados del CdA. Los cálculos estratégicos de los Estados del golfo, vistos como un todo, implican sus rivalidades en regiones como Medio Oriente y también se trasladan a su competencia en el $\mathrm{CdA}$, en este sentido, los cambios de política exterior de Sudán se explican por la rivalidad iraní-saudí, al igual que en el caso de Soma- lia, sus políticas de consolidación interna se encuentran condicionadas por la influencia de Turquía-Qatar contra EAU, como ejemplos puntuales.

\section{La estrategia de inserción desde el punto de vista de los Estados del CdA}

La importancia creciente del CdA ha otorgado una ventana de oportunidad a los Estados de la región para modificar sus situaciones internas y relaciones interestatales. Este escenario favorable se caracteriza por la conjunción de una mejora considerable de los índices de crecimiento económico, atracción de inversiones directas, geopolítica regional y posicionamiento estratégico, que incentivan un mayor acercamiento de nuevos actores con capacidades militares, económicas, financieras y diplomáticas quienes ofrecen sus recursos para modificar el contexto regional (ADBG, 2018).

En este sentido la interacción de intereses locales y extra-regionales establecerá mecanismos de negociación, competencia, reasignaciones y demandas que modificarán sustancialmente el escenario estratégico regional. Los Estados del CdA contarían con un margen de maniobra mayor, gracias a la multiplicidad de opciones disponibles en las que se incluyen la presión por condiciones de acceso favorables y la potencialidad de actuar en forma coordinada contra el creciente flujo de políticas de inserción estratégica.

En relación con esto último, se pueden analizar las capacidades de acción de los Estados del CdA como actores activos y con un poder de influencia para definir el proceso de inserción de otros Estados en la región, dado que pueden 
establecer agendas de tipo local, que interpretan este nuevo contexto de mayor intervención extranjera como favorable para el alcance de ciertos objetivos estratégicos propios, de mediano y largo plazo, como veremos a continuación.

Eritrea ha sido un país aislado diplomáticamente y con un conflicto limítrofe con Etiopía que estalló con la guerra entre 19982000. La definición de sus fronteras territoriales y la adquisición de aliados diplomáticos han orientado su política estratégica. Los esfuerzos de guerra, la leva masiva y la precariedad socioeconómica han potenciado los abusos de derechos humanos y la migración masiva hacia otras regiones (HRW, 2019). Su situación cambió drásticamente con la guerra en Yemen, su cercanía litoral, adecuada para la creación de bases militares le otorgó un nuevo rol primordial en el escenario de guerra. Renunciando a sus relaciones amistosas con Irán, recibió de EAU y Arabia Saudita apoyos financieros, militares y diplomáticos que aliviaron su precaria situación económica y quienes, a su vez, apoyaron la búsqueda de pacificación de sus diferendos con Djibouti y Etiopía. En la actualidad, la presencia oficial de dirigentes rusos y una cercanía más reticente de los EE. UU. han potenciado el interés diplomático en Eritrea quien, a su vez, busca en estas nuevas oportunidades acceder a inversiones, promover la creación de infraestructuras y mejorar sus vínculos diplomáticos, aunque esto implique un alineamiento con países como Arabia Saudita, el rechazo de relaciones amistosas históricas con Irán o su involucramiento indirecto en un conflicto bélico.

Etiopía es uno de los actores más relevantes a nivel regional como ya hemos mencionado. Su capacidad económica, basada en la mano de obra accesible y recursos naturales, ha atraído diversas inversiones extranjeras que han potenciado su crecimiento económico (World Bank, 2019). La política del ministro Ahmed, orientada a imprimir un rol activo y diplomático más ambicioso de su país, ha llevado al apoyo a la pacificación territorial con Eritrea, aumentando la cooperación con Djibouti principal salida de sus productos al exterior-a su vez, ofició como moderador en el conflicto armado de Sudán del Sur y con intereses especiales en la transición política de Sudán. El crecimiento económico ha requerido de la creación de infraestructuras logísticas, zonas comerciales industrializadas y empresas energéticas que han generado tensiones en relación con el problema de la asignación de recursos (International Crisis Group, 2019). Problemas internos han sido aquellos vinculados a las oposiciones étnicas a causa de la distribución de ingresos, como ha sido la crisis de Tigray en noviembre de 2020, el daño ambiental y la reasignación de tierras, mientras que, en el ámbito externo, los desafíos se vinculan a los proyectos hidroeléctricos, siendo representativo el asunto de la represa del Renacimiento, sobre el cauce alto del Nilo, que afectaría el uso compartido de aguas con Sudán y Egipto, aliado principal de los saudíes y quienes han apoyado financieramente al gobierno etíope.

La política exterior etíope se encuentra potenciada y limitada por el juego relacional de otros actores estratégicos con intereses específicos en la región. Su creciente cooperación con Djibouti está vinculada a las inversiones chinas quienes tienen en Etiopía sus principales inversiones industriales y compromisos de deuda y que requieren de nuevos medios para 
retirar la producción por un puerto controlado y seguro como es el de Djibouti, en donde actualmente han creado varias vías férreas, eléctricas, energéticas y de transporte entre ambos países al tiempo que cuentan con una base militar y portuaria. En este ejemplo, se puede interpretar que el rol económico creciente de Etiopía necesita y promociona la intervención extranjera, pero que en el mediano plazo condiciona sus opciones estratégicas de cooperación e inserción global.

Somalia es otro actor interesante para observar la dinámica en cuestión, claramente representada en las tensiones entre el gobierno central somalí, ubicado en Mogadiscio, y el gobierno de Putland y Somalilandia, quienes actúan de facto como un gobierno separado. El gobierno central ha sido el producto de los esfuerzos internacionales destinados, desde 1991 hasta la fecha, a la reconstrucción de un Estado. Las guerras civiles, el terrorismo de Al-Shabbab y la invasión etíope de 2007 convirtieron al territorio central en un espacio en disputa. La política del Departamento de Estado de EE. UU., al igual que la de la Amisom y la UA, permitieron la recuperación territorial del Estado (Burgess, 2015). En la actualidad, las políticas de acercamiento de Turquía y en segundo lugar de Qatar han representado un increíble esfuerzo de reconstrucción comercial, habitacional, logística y de servicios que han permitido la proyección del poder central sobre el interior del país.

Las regiones norteñas de Puntland y Somalilandia han sido menos afectadas por los procesos previamente mencionados y han gozado de la existencia de un gobierno sostenido y coherente, potenciando el crecimiento económico y la estabilidad institucional de forma separada del gobierno central. A fin de garantizar su soberanía, el gobierno de Somalilandia ha desplegado una política exterior autónoma que se ha beneficiado de las necesidades estratégicas vinculadas al control de la piratería en el golfo de Adén. Por otro lado, la guerra en Yemen ha repercutido en el desembarco de nuevos actores como EAU quienes, ante su desplazamiento de Djibouti, han reorientado sus intereses de cooperación con estas regiones vecinas ofreciendo inversiones portuarias, logísticas y de acceso financiero solo viables gracias a la posición geoestratégica, beneficios de privilegio que se perderían si estuvieran sometidos a las necesidades políticas del gobierno central.

Finalizando con el caso de Djibouti, se debe considerar que ha sido el país donde la vinculación de intereses estratégicos extraregionales con los nacionales ha convergido, de manera más intensa, y que presentan un carácter histórico. En este aspecto, la posición de Djibouti, en el punto más estrecho que conecta el Bab el Mandeb con el mar Rojo, le otorga una relevancia importantísima compartida solo con Yemen, país que se encuentra en guerra civil.

Djibouti es reconocido por ser el país con mayor cantidad de bases militares extranjeras instaladas en territorio nacional en el mundo 5 . La economía de Djibouti depende estricta-

5 Francia, Italia, Japón, EE. uU., Arabia Saudita, China y hay una invitación para una base de Turquía. 
mente de sus ingresos portuarios, claves en el cruce de pasos estratégicos como los antes mencionados y uno de los más desarrollados de la región del CdA. A su vez, su posición privilegiada y con escasa competencia cercana le vuelve un punto de tráfico central para la salida de existencias de Etiopía, uno de los principales motores económicos de la región. Dado que el país no cuenta con recursos estratégicos y naturales de privilegio, las regalías comerciales y la tercerización de la protección portuaria son sus principales recursos económicos y diplomáticos, mediante los cuales, el otorgamiento de concesiones portuarias o la obtención de privilegios de permanencia y comercial, le permiten a Djibouti contar con una gran capacidad de maniobra pese a su limitada capacidad en recursos financieros, militares y diplomáticos (ICG, 2019, p. 5). En este aspecto, es un caso paradigmático y extremo de la fenomenología extendida a la región del CdA que ejemplifica la capacidad de acción de un Estado adquirida por la pluralidad de intereses estratégicos en un espacio compartido.

\section{CONCLUSIONES}

A lo largo del trabajo se han comentado los elementos que otorgan una importancia estratégica al CdA en la actualidad, desde su posicionamiento geopolítico, la existencia de una gran diversidad de intereses y actores y la complejidad estratégica generada por este escenario.

Aunque las implicaciones estratégicas en la región se encuentran repartidas entre una gran cantidad de actores con diferentes grados de poder, se destacan aquellos que utilizan el escenario como parte de su proyección global y otros que se acercan al mismo como extensión de sus problemáticas regionales, generando un proceso que denominamos traslación de conflictividad, como hemos observado en el caso de Turquía y Eau en Somalia o Arabia Saudita, e Irán, en Eritrea y Sudán.

En este último aspecto, el papel de los denominados países del golfo (Arabia Saudita, EAU y Qatar) a los que se agrega Irán y Turquía juegan un rol fundamental en este proceso de traslación de conflictividad que generan, de forma más directa, un entramado de beneficios y riesgos para la estabilidad y desarrollo regional.

A su vez, hemos destacado que la existencia de esta situación no se puede explicar sin el papel protagónico de los Estados africanos del CdA. Estos buscan ventajas en función del creciente interés en la región, indagando la consecución de sus intereses estratégicos específicos pese a que impliquen limitaciones y potencialidades, al tiempo que redefinen los alcances y medios disponibles para las políticas de inserción extra-regionales. En este sentido, la creciente importancia de Eritrea y su extenso litoral en el mar Rojo, le ha permitido contar con un poder de negociación para definir sus relaciones con Estados como Irán, EAU y Arabia Saudita, entre otros.

Por lo tanto, se observa que la inserción estratégica en el CdA no se puede explicar con un abordaje pasivo de los actores del litoral africano, al mismo tiempo que la interacción entre estos y aquellos Estados con intereses globales y regionales se define por una constante readaptación de políticas y estrategias desplegadas, las cuales afectan las agendas internas y 
externas de los actores y que requieren de un seguimiento paulatino e interpretativo debido a su condicionamiento por una coyuntura de elevada volatilidad.

\section{REFERENCIAS}

Asya, A. (2019). Neo-ottomanism: Turkey's foreign policy approach to Africa, Center for Strategic \& International Studies [Csis], New perspectives in foreign policy, issue 17, April. Disponible en https:// www.csis.org/neo-ottomanism-turkeys-foreignpolicy-approach-africa

African Development Bank Group. (2018). Investing in transition states: the horn of Africa opportunity. Disponible en https://www.afdb.org/fileadmin/ uploads/afdb/documents/generic-documents/investing_in_transition_states-_the_horn_of_africa_opportunity_-_a_briefing_note_for_south_ korean_investors.pdf

Amin, M. (19/03/2018). Suakin: 'Forgoten' Sudanese island becomes focus for Red Sea rivalries. Middle East Eye. Disponible en http://www.middleeasteye.net/news/suakin-island-sudan-turkey-saudiarabia-egypt-394055164

Burgess, S. (2015). The United States in the Horn of Africa. The role of the military, Aspj Africa \& Francophonie, $1^{\text {st }}$ quarter, pp. 27-36. Disponible en https://www.airuniversity.af.edu/Portals/10/ ASPJ_French/journals_E/Volume-06_Issue-1/ burgess_e.pdf

Encyclopedia Britannica. (2021). Horn of Africa. Disponible en https://www.britannica.com/place/ Horn-of-Africa

European Center for Development Policy Management. (2021). Regional Organisations in Africa. Disponible en https://indd.adobe.com/view/f49ac87d7aa3-4cf7-822e-841d674bbc92
Gurbuz, M. (2018). Turkey challenge to Arab interest in the horn of Africa. Arab Center, Washington DC, February. Disponible en http://arabcenterdc. org/policy_analyses/turkeys-challenge-to-arabinterests-in-the-horn-of-africal

Gulf Business. (1/05/2018). Gulf rivals battle for ports access in Somalia, May, Disponible en https:// gulfbusiness.com/gulf-rivals-battle-for-portsaccess-in-somalia/

Human Right Watch. (2019). Eritrea, Events of 2018. World Report, Disponible en https://www.hrw. org/world-report/2019/country-chapters/eritrea

IGAD. (2019). What we do. Disponible en https://igad. int/about-us/what-we-do

International Crisis Group. (2019). Intra-gulf competition in Africa's horn: lessening the impact. Middle East Report No. 206, September, p. 9. Disponible en https://www.crisisgroup.org/middle-east-northafrica/gulf-and-arabian-peninsula/206-intragulf-competition-africas-horn-lessening-impact

International Crisis Group. (2019). Ethiopia: managing Ethiopia's unsettled transition. Report No. 269, February. Disponible en https://www.crisisgroup. org/africa/horn-africa/ethiopia/269-managingethiopias-unsettled-transition

Institut de Relations Internationales et Stratégiques. (2017). East Africa and the horn in 2022. An Outlook for Strategic Positioning in the Region. [IRIs], March. Disponible en https://www.iris-france. org/wp-content/uploads/2017/03/овs-Prospective-huma-East-Africa-Horn-mars-2017.pdf Isern, P. (2021). Relaciones internacionales y juegos de suma cero, Fundación ICBC. https://www.biblioteca.fundacionicbc.edu.ar/index.php/Relaciones_Internacionales_y_juegos_de_suma_cero

Iss. (2019). Peace Security Council Report. Disponible en https://issafrica.s3.amazonaws.com/site/uploads/ pscreport112.pdf 
Lefebvre, J. (2012). Iran in the Horn of Africa: Outflanking U.S allies. Middle East Policy Council, vol. xIx, No. 2. Disponible en https://mepc.org/ journal/iran-horn-africa-outflanking-us-allies

Leboeuf, A. (2019). La competition stratégique en Afrique: approches militaires américaine, chinoise et russe. Études de lifri, focus Stratégique. Disponible en https://www.ifri.org/sites/default/ files/atoms/files/leboeuf_competition_strategique_afrique_2019_0.pdf

Lin, J. Y. (20/01/2015). Industry transfer to Africa good for all. China Daily. Disponible en https://www. chinadaily.com.cn/opinion/2015-01/20/content_19353077.htm

Mahmood, O. (2019). Competition, cooperation and security in the Red Sea. Institute of Security Studies [Iss]. East Africa Report No. 24, August. Disponible en https://issafrica.org/research/ east-africa-report/competition-cooperation-andsecurity-in-the-red-sea

Mahmood, O. (2019). How the Gulfcrisis is destabilizing Somalia. Institute of Security Studies [Iss], October. Disponible en https://issafrica.org/iss-today/ how-the-gulf-crisis-is-destabilising-somalia

Marchal, R. (2018). Mutations géopolitiques et rivalités d'Etats: La Corne de l'Afrique prise dans la crise $\mathrm{du}$ Golfe. Observatoire Afrique de l'est. Marzo. Disponible en https://www.sciencespo.fr/ceri/fr/content/mutations-geopolitiques-et-rivalites-d-etatsla-corne-de-l-afrique-prise-dans-la-crise-du-go

Marchal, R. (2020). Quatre questions sur le Soudan, un an après. SciencesPo. Disponible en https://www. sciencespo.fr/ceri/fr/content/soudan-un-apresentretien-avec-roland-marchal.html

Mesfin, B. (2019). Could Emirati activism in the Horn make the situation worse. Institute of Security Studies [Iss], October. Disponible en https://issa- frica.org/amp/iss-today/could-emirati-activismin-the-horn-make-the-situation-worse

Neil, J. (2019). External security politics of the Horn of Africa region. Stockholm International Peace Research Institute [SIPRI]. Insights on peace and security $n^{\circ}$. 2019/2. Disponible en https://sipri. org/news/2019/sipri-launches-reports-new-external-security-politics-horn-africa-region

Ouma, S. (2012). The New Scramble for Africa, Regional Studies, 46:6, 836-838, Doi: 10.1080/00343404.2012.691237.

Reuters. (17/01/2017). Somalia received sandi aid the day it cut ties with Iran: document. January. Disponible en http://www.reuters.com/article/us-somaliasaudi-iran-iduskcn0uv0bh

Scholvin, S. (2016). Geopolitics. An Overview of concepts and empirical examples from international relations. The Finnish Institute of International Affairs. Disponible en https://www.files.ethz.ch/ isn/196701/wp91-Geopolitics.pdf

Skinner, A. (22/01/2021). End of the Qatar Embargo: An Opportunity, not Threat, for Turkey. Washington Institute. Disponible en https://www.washingtoninstitute.org/policy-analysis/end-qatar-embargo-opportunity-not-threat-turkey

The Maritime Executive. (04/05/2019). Djibouti Ordered to Pay DP World \$530M in Port Dispute. Disponible en https://www.maritime-executive.com/ article/djibouti-ordered-to-pay-dp-world-530min-port-dispute

Ursu, A.-E. \& Van den Berg, W. (2018). China and the $E U$ in the horn of Africa: competition and cooperation? Clingendael Institute, Cru policy brief, Abril. Disponible en https://www.clingendael. org/sites/default/files/2018-04/PB_China_and_ the_EU_in_the_Horn_of_Africa.pdf 
United States Agency for International Development. (2021). Somalia. Usaid. Disponible en https:// www.usaid.gov/somalia

Van den Berg, W. \& Meester, J. (2019). Turkey in the Horn of Africa. Between the Ankara consensus and the Gulf crisis, Clingendael Institute May. Disponible en https://www.clingendael.org/ sites/default/files/2019-05/PB_Turkey_in_the_ Horn_of_Africa_May_2019.pdf

Woldemichael, S. \& Andemariam, S. (2019). Can the peace-trade equation stabilize the horn. Institute of
Security Studies [ISS] July. Disponible en https:// issafrica.org/iss-today/can-the-peace-trade-equation-stabilise-the-horn

Woldemichael, S. (2020). Sudan's transition. What are the chances of success? Institute of Security Studies [Iss]. East Africa Report No. 35. November. Disponible en https://issafrica.s3.amazonaws.com/ site/uploads/ear-35.pdf

World Bank. (2019). Disponible en https://www.worldbank.org/en/country/ethiopia/overview 